\title{
Review
}

\section{Advances in Research on the Role of Chemokines in Occurrence and Development of Autoimmune Thyroid Disease}

Guang Ji

Department of Internal Medicine, Hospital of Hebei University of Technology, Tianjin China

\author{
Keywords \\ Chemokine; Hashimoto's thyroiditis; Graves' \\ disease; Autoimmune thyroid disease

\section{Correspondence} \\ Guang Ji, \\ E-mail: jghebut@126.com \\ DOI: 10.1515/ii-2017-0108
}

\begin{abstract}
Chemokines can be divided into four categories: $\alpha, \beta, \gamma$, and $\delta$. Chemokine $\alpha$ is related to neutrophil chemotaxis. Chemokine $\beta$ is correlated with adsorption of monocytes, basophils, and eosinophils. Chemokine $\gamma$ is mainly a lymphocyte chemokine. Function of chemokine $\delta$ remains unclear. Chemokines $\alpha$ and $\beta$ are primarily related to occurrence and development of autoimmune thyroid disease. This study reviews chemokines and their receptors that are related to Graves' disease and Hashimoto's thyroiditis.
\end{abstract}

Autoimmune thyroid diseases (AITD) include Graves' disease (GD) and Hashimoto's thyroiditis (HT). The former takes advantage of stimulation of thyroid function, whereas the latter destroys thyroid tissues. However, pathogenesis of these disease still require full understanding. Chemokines play important roles in many autoimmune diseases, such as rheumatoid arthritis, myasthenia gravis, and multiple sclerosis. Thus, in recent years, considerable attention was paid to structure, function, and effects of chemokines and their receptors on AITD pathogenesis ${ }^{[1-6]}$. This study discusses chemokines and their receptors that are closely related to pathogenesis of AITD based on the following aspects: definition of chemokines, chemokines and their receptors that are associated with AITD, and chemokines and their receptors that are related to GD and HT.

\section{Overview of chemokines associated with AITD}

\section{Chemokine}

Chemokines represent a group of secretory proteins whose relative molecular mass measures 8000 to 12,000 . Chemokines can activate leukocyte chemotaxis, attract neutrophils and monocyte/macrophages to inflammation lesions, enhance phagocytosis of inflammatory cells, and promote release of inflammatory proteins and inflammatory mediators. Chemokines also directly participate in inflammatory processes. Cytokines and chemical inducers can regulate chemokines. In the immune system, interleukin (IL), interferon (IFN), tumor necrosis factor (TNF), and transforming growth factor-beta (TGF- $\beta$ ) interact with each other, play important regulatory functions, and in abnormal situations, cause immune pathological reactions. According to position of conserved cysteine (C), structural chemokines can be divided into the following: (1) chemokine $a$ (chemokine (C-X-C motif) ligand 1 (CXCL1)-17), which is related to neutrophil chemotaxis; (2) chemokine $\beta$ (CCL1-28), which is associated with adsorption of monocytes, basophils, and eosinophils; (3) chemokine $\gamma$ (XCL1 and XCL2), which is a lymphocyte chemokine; and (4) chemokine $\delta(\mathrm{CX} 3 \mathrm{C} 1)$, which anchors only to cell membrane of the brain. Chemokine receptors belong to seven transmembrane $\mathrm{G}$ protein-coupled receptor superfamily, which can be divided into CXC, CC, C, and $\mathrm{CX} 3 \mathrm{C}$ receptors. Cells with immune characteristics and endothelial cells mainly express chemokine receptors. Factors and receptors show diverse interaction. Chemokines can bind to many receptors. For example, chemokine Regulated Upon Activation of Normal T-cell Expressed and Secreted (RANTES) can bind to CCR (1, 3, and 5) and three receptors. In the same sense, one receptor can also combine with different chemokines. The receptor CCR3 can bind to chemokine monocyte chemotactic protein (MCP)-22, 
eotaxins, and MCP-23. Studies on chemokines and their receptors made advances considering that some chemokines were found to inhibit HIV infection in 1995. To date, nearly 50 chemokines and 20 chemokine receptors are discovered.

\section{Chemokines and receptors associated with AITD}

In recent years, AITD-related chemokines became a significant research topic. Some studies showed ${ }^{[7]}$ that mRNA expression of CXC subtribe chemokine IFN induced protein- $\gamma$-10 (IP-10) and IFN- $\gamma$-induced mononuclear cell factor (Mig) in GD and HT thyroid tissue samples. Goulvestre et al. ${ }^{[8]}$ studied cytokines and chemokines expressed in thyroid tissues. Their results showed increased expressions of CCL21, CXCL12, CXCL13, and CCL22 in thyroid tissues of AITD patients. Especially, significant increases were observed in expressions of CXCL12, CXCL13, and CCL22 formed by ectopic lymphoid follicles in AITD tissues. B-Cell expression in type 1 diabetic patients can induce IFN- $\gamma$ to produce chemokine CXCL9 and 10, which are associated with CXCR3-positive cytotoxic T cells entering islets of Langerhans ${ }^{[9]}$. Kemp et al. ${ }^{[10]}$ discovered that factors, such as MCP-1, expression of normal T cells and secretory factor, and macrophage inflammatory protein-1 $\alpha$, are expressed in thyroid gland tissues of most GD patients. Reverse transcription polymerase chain reaction presented abnormal expression of IFN- $\gamma$ transgenic mice, which were used as HT model, and results indicated expression of CCL4, CXCL9, and CXCL11 ${ }^{[11]}$. Some studies ${ }^{[12]}$ proved that CCL2 is related to pathogenesis of experimental autoimmune encephalomyelitis.

\section{Chemokines associated with GD and related studies}

\section{Chemokines associated with GD}

Studies conducted in 1992,1993, and 1999 showed higher primary cultured thyroid cells and in-vitro-cultured peripheral blood mononuclear cells and peripheral blood CXCL8 of GD patients than normal individuals. Levels of CCL3, CXCL12, CCL21, and CCL4 mRNA in thyroid of GD patients were higher than those of patients with nodular goiter. Factors associated with GD include CXCL13 and its receptor, CXCR5 and CCL5. In recent years, studies on the relationship between chemokines and GD mainly centered on chemokines CXCL 9 and $10^{[13,14]}$.

\section{Hotspot chemokines associated with GD}

IP-10 and Mig are mainly concentrated in GD thyroid follicular epithelial cells (TFCs), which are adjacent to lymphocytic infiltration ${ }^{[15]}$. IP-10 and Mig expressions in thyroid tissues are affected by various proinflammatory cytokines, of which IFN- $\gamma$ is the most important. In GD, lymphocytes activated by autoimmunity secrete large amounts of proinflammatory cytokines, such as IFN- $\gamma$, providing environmental conditions for induction and composition of IP-10 and Mig. Then, IP-10 and Mig chemotaxis of inflammatory cells (mainly $\mathrm{T}$ lymphocytes) infiltrate, proliferate, induce immune reaction mediated by specific antigens, and destruct the thyroid gland or its functions. Activated T lymphocyte secrete various cytokines, including TNF- $\alpha$, IFN- $\gamma$, and IL, inducing expression of major histocompatibility antigen complex-class II antigen in TFC of lesions. This phenomenon enhances autoantigen capacity of macrophages and dendritic cells. Simultaneously, intercellular adhesion molecule-1 and lymphocyte functionrelated antigen-3 play important roles in recognition of antigenic specificity, hyperplasia-related signal transduction, and effector cells on target cells. During IFN- $\gamma$-induced IP-10 and Mig formation, cytokines TNF- $\alpha$ and IL-1 $\beta$ exhibit strong synergistic effects. IL-4 can increase induced effects of IFN- $\gamma$ and TNF- $\alpha$ on IP-10 and Mig. Garcia-Lopez et al. observed that TFCs cultured in vitro of AITD patients expressed IP-10 and Mig, and these expressions increased after stimulation of TFCs by IFN- $\gamma$. Compared with peripheral blood lymphocytes, expressions of chemokine receptor CXCR3, CCR2, and CCR5 increase in thyroid gland lymphocytes of AITD patients. These results illustrate that TFCs can regulate specific types of lymphocyte recruitment during AITD formation by generating chemotactic factors, such as IP-10 and Mig. High level of CXCL10 in serum is related to active markers of autoimmune diseases. Corona et al. ${ }^{[16]}$ discovered that blood flow in thyroid gland increases when level of CXCL10 significantly increased in the peripheral blood of patients with GD. Maximal systolic velocity and thyroid volume of thyroid gland are correlated with levels of CXCL10 in peripheral blood. This phenomenon indicates that thyroid angiogenesis is regulated by CXCL10 effect. Several angiogenesis events were observed during inflammation; these phenomena occurred upon reaching a relatively balance point between angiogenesis factors and stability factors ${ }^{[17]}$. Occurrence and changes in thyroid new blood vessels in patients with GD, 
which is characterized by predominance of thyroid function, are related to high concentration of vascular endothelial growth factor generated by TFCs, which are involved in thyroid-stimulating antibody response. After CXCR3 montage, combination of variant B and CXCL10 stabilizes angiogenesis. However, angiogenic factors can completely destabilize variant $\mathrm{B}$.

\section{Chemokines associated with HT and related studies}

\section{Characteristics of $\mathrm{HT}$}

HT is characterized by specific thyroid autoantibodies, such as antithyroperoxidase antibody (TPOAb) and antithyroglobulin antibody (TGAb), in vivo and infiltration of a large number of inflammatory cells in the thyroid gland; it severely destroys normal structure of the thyroid and decreases thyroid function. HT pathogenesis remains to be clarified. However, scholars consider possible disruption in balance between inhibitory $\mathrm{T}$ lymphocytes and auxiliary $\mathrm{T}$ cells. Function of the former decreases, whereas that of the latter increases. This phenomenon enables B lymphocytes, the most common of which are TPOAb (previously known as anti-thyroid microsome antibody) and TGAb, to produce anti-thyroid autoantibodies in large quantities. Cytotoxicity mediated by some of these antibody cells and natural killer cells damages thyroid cells, and thyroid tissue is infiltrated by inflammatory cells, mainly by lymphocytes and CD4+ $\mathrm{T}$ cells; a small part of CD8+ cytotoxic T lymphocytes also participates in this phenomenon ${ }^{[18]}$.

\section{Chemokines associated with HT}

Thyroid of HT patients present increased expression levels of CCL3, 4, and 5 and CXCL12, CXCL13, CXCL14, CCL18, CCL19, CCL21, and CCL22. An increase can also be observed in expression levels of chemokine CXCL9 and 10 , which can induce IFN- $\gamma$ in thyroid of HT patients. Combination of HT chemokines CXCL10 and CXCR3 mainly recruits activated $\mathrm{T}$ lymphocytes. Then, lymphocytes and other inflammatory cells are activated and diffusely infiltrate thyroid tissues. Some studies indicated that CXCL10 plays important pathological roles during the initial stage of HT; these functions include expression of CXCL10 in the newly formed vascular endothelial cells and infiltration of inflammatory cells and thyroid cells. Expression site of CXCR3 receptor is also consistent. No expression of
CXCL10 was detected in glands of non-AITD patients. High expression of CXCL10 in thyroid cells obtained from HT patients was observed by immunohistochemistry. By evaluating mRNA and protein levels in glands, high expression of CXCL10 confirmed the role of CXCL10 in HT [19-21].

\section{Study model of chemokines associated with HT}

To investigate roles of chemokines in HT, most researchers combined usage of thyroglobulin and immune adjuvant through injection in animals, mainly rabbit HT models. Thyroid glands of immune animal can express CCL2 (MCP-1) and CCL5 (RANTES). Infiltration of inflammatory cells in the thyroid usually lasts for two months, and normal structure of thyroid gland recovers after this incident. This situation prevents animal models from simulating actual chronic thyroid destruction in human HT patients. Mice lymphocytes immunized with $\mathrm{Tg}$ and isolated and cultured by thyroglobulin and IL-12 in vitro are injected into normal mice to induce intra-thyroid granulomatous inflammation and to cope with deficiency of rabbit HT model. Application of IFN- $\gamma$ transgenic mouse model positively affects studies of the relationship between chemokines and HT.

In summary, chemokines play important roles in pathogenesis of AITD. Monitoring changes of chemokines in peripheral blood of patients with AITD serves as reference for evaluating and predicting development of AITD. In-depth clinical and basic experimental studies enable detection of serum chemokine changes ${ }^{[22,23]}$. Related products of chemokines and their receptors are important topics for clinical research, which are expected to bring new diagnostic and therapeutic methods for AITD, organ-specific autoimmune diseases, and tumors ${ }^{[24-30]}$. Pathogenesis of AITD remains to be clarified. Epidemiological investigation and animal models confirmed that excessive intake of iodine can induce or aggravate AITD occurrence and progression. However, further research should still elucidate pathogenic mechanism of iodine on AITD. Additional studies should also determine whether expression of chemokines and their receptors is the key link in excess iodine in the thyroid body. Research should also focus on actively exploring pathogenesis of AITD and identifying specific mechanism of its occurrence and development to determine specific intervention methods. 


\section{Declarations}

\section{Acknowledgements}

No.

\section{Competing interests}

The author declares that she has no competing interest.

\section{Authors' contributions}

G Ji made the literature analysis and wrote, discussed and revised the manuscript of this review.

\section{References}

1 Smith TJ, Padovani-Claudio DA, Lu Y, et al. Fibroblasts expressing the thyrotropin receptor overarch thyroid and orbit in Graves'disease.J Clin Endocrinol Metab, 2011, 96(12):3827-3837.

2 Polymeris A, Karoutsou E, Doumouchtsis K. Seronegative Myasthenia Gravis and Graves'Disease.Is there a link?. Exp Clin Endocrinol Diabetes, 2012, 120(5):254-256.

3 Antonelli A, Ferri C, Ferrari SM, et al. Interleukin- $1 \beta, \mathrm{C}-\mathrm{X}-\mathrm{C}$ motif ligand 10 , and interferon-gamma serum levels in mixed cryoglobulinemia with or without autoimmune thyroiditis. J Interferon Cytokine Res, 2010, 30(11): 835-842.

4 Antonelli A, Ferrari SM, Frascerra S, et al. Circulating CXCL9 is increased in aggressive chronic autoimmune thyroiditis,in association with CXCL10. Cytokine, 2011, 55(2):288-293.

5 Antonelli A, Ferrari SM, Frascerra S, et al. Increase of circulating CXCL9and CXCL11associated with euthyroid or subclinically hypothyroid autoimmune thyroiditis. J Clin Endocrinol Metab, 2011,96(6):1859-1863.

6 Dong QY, Li SJ, Gao GQ et al. Short-term effect of radioactive iodine therapy on CXCL-10production in Graves' disease.Clin Invest Med, 2011,34(5):E262.

7 Garcia-Lopez MA, Sancho D, Sanchez-Madrid F, et al. Thyrocytes from autoimmune thyroid disorders produce the chemokines IP-10 and Mig and attract CXCR3+lymphocytes. J Clin Endocrinol Metab, 2001,86(10): 5008-5016.

8 Goulvestre C, Batteux F, Charreire J, et al. Chemokines modulate experimental autoimmune thyroiditis through attraction of autoreactive or regulatory T cells. Eur J Immunol, 2002,32(12):3435-3442.

9 Frigerio S, Junt T, Lu B, et al. Beta cells are responsible for CXCR3mediated T-cell infiltration in insulitis. Nat Med, 2002,8(12):1414-1420. of chemokine gene expression in autoimmune thyroid disease. Clin Endocrinol (Oxf), 2003,59(2):207-213.

11 Kimura H, Kimura M, Rose NR, et al. Early chemokine expression induced by interferon-gamma in a murine model of Hashimoto's thyroiditis. Exp Mol Pathol, 2004,77(3):161-167.

12 Ubogu EE, Cossoy MB, Ransohoff RM. The expression and function of chemokines involved in CNS inflammation. Trends Pharmacol Sci, 2006, 27(1):48-55.

13 Domberg J, Liu C, Papewalis C, et al. Circulating chemokines in patients with autoimmune thyroid diseases. Horm Metab Res, 2008,40(6):416-421.

14 Leite AC, Pedro AB, Romaldini JH. Influence of methimazole and radioactive iodine treatment in the serum levels of the chemokine CXCL10in hyperthyroid patients with Graves' disease. Horm Metab Res, 2011,43(3):194-199.

15 Antonelli A, Rotondi M, Fallahi P, et al. Increase of interferongamma inducible alpha chemokine CXCL10but not beta chemokine CCL2serum levels in chronic autoimmune thyroiditis. Eur J Endocrinol, 2005,152(2):171-177.

16 Corona G, Biagini C, Rotondi M, et al. Correlation between, clinical, biochemical, color Doppler ultrasound thyroid parameters, and CXCL10in autoimmune thyroid diseases. Endocr J, 2008,55(2):345-350.

17 Romagnani P, Lasagni L, Annunziato F, et al. CXC chemokines: the regulatory link between inflammation and angiogenesis. Trends Immunol, 2004,25(4):201-209.

18 Sospedra M, Ferrer-Francesch X, Dominguez O, et al. Transcription of a broad range of self-antigens in human thymus suggests a role for central mechanisms in tolerance toward peripheral antigens. J Immunol,1998,161(11):5918-5929.

19 Itoh K, Meffre E, Albesiano E, et al. Immunoglobulin heavy chain variable region gene replacement as a mechanism for receptor revision in rheumatoid arthritis synovial tissue B lymphocytes. J Exp Med,2000,192 (8):1151-1164.

20 Cakir M, Levendoglu F, Kiyici A, et al. Serum CXCL10 levels and neuromuscular manifestations in patients with autoimmune thyroid diseases. Autoimmunity, 2011, 44(6):496-503.

21 Ansel KM, Ngo VN, Hyman PL, et al. A chemokine-driven positive feedback loop organizes lymphoid follicles. Nature, 2000, 406(6793):309-314.

22 Gillespie EF, Smith TJ, Douglas RS. Thyroid eye disease: towards an evidence base for treatment in the 21st century. Curr Neurol Neurosci Rep, 2012, 2012, 12 (3) :318-324.

23 Rotondi M, Chiovato L. The chemokine system as a therapeutic target in autoimmune thyroid diseases:a focus on the interferon$\gamma$ inducible chemokines and their receptor. Current Pharmaceut Design, 2011,17(29):3202-3216.

24 Bozec A, Lassalle S, Hofman V, et al. The thyroid gland:a crossroad in inflammation-induced carcinoma ?An ongoing debate with new therapeutic potential. Curr Med Chem, 2010,17(30):3449-3461.

25 O'Neal R. CCR5inhibitors: up and coming new agents. BETA, 
2007,19(2):15-19.

26 Hogaboam CM, Bone-Larson C, Matsukawa A, et al. Therapeutic use of chemokines. Current Pharmaceutical Design, 2000, 6 (6) :651-63

27 Fang Y,L Zhao L,Yan F. Chemokines as novel therapeutic targets in autoimmune thyroiditis. Recent Patents on Dna \& Gene Sequences, 2010, 4 (1):52-7

28 Leone V, D'Angelo D, Rubio I, et al. MiR-1 is a tumor suppressor in thyroid carcinogenesis targeting CCND2, CXCR4, and SDF-1alpha. J
Clin Endocrinol Metab, 2011, 96(9):E1388-1398.

29 N Mach, G Dranoff. Cytokine-secreting tumor cell vaccines. Current Opinion in Immunology, 2000, 12(5):571-575.

30 N Akeno, EP Smith, M Stefan, et al. IFN- $\alpha$ mediates the development of autoimmunity both by direct tissue toxicity and through immune cell recruitment mechanisms. Journal of Immunology, 2011, 186(8):4693-4706. 